\title{
Inverse variational principle based coupled modeling of underground structures
}

\author{
P. Procházka \\ Czech Technical University in Prague, Czech Republic
}

\begin{abstract}
In some previous papers of the author coupled numerical-experimental modeling of tunnels embedded in surrounding rock was based on minimization of a certain functional describing the steepest descend mode of differences of measured and computed values of stresses or displacements at selected points. The idea consisted of a choice of subdomains (patches), the eigenstrains in which were introduced using the unit impulse technique. Influence matrices were generated and the linear hull of eigenstrain effects together with the optimization problem lead to the identification of plastic stresses inside the domain describing the surrounding rock. Consequently, a nonlinear model in numerical analysis can be improved using eigenparameters as design parameters in optimization. The only problem remaining is how to select the patches. In this paper inverse variational principles are applied to help solve this principal problem. The 2D problem is solved with moving patches (support subdomains) with uniformly introduced eigenstrains.

Keywords: coupled modeling, tunnel face stability, Inverse variational principles, Desai's model.
\end{abstract}

\section{Introduction}

Using a very powerful tool, a combination of Transformation field analysis (TFA) and a certain plasticity rule (possibly softening included), back analysis of structures can be regarded as seeking the optimal distribution of eigenparameters (eigenstrains or eigenstresses) in the domain describing undeformed rock in a certain sense. For the first time the TFA was applied to optimization of prestressing of composite structures, [1]. The eigenparameters can represent many phenomena (not only prestress), and also the influence of plastic 
deformation, or the influence of the length of purchase in tunneling. A similar approach was applied to nonlinear problems in composite structures, [2]. In the first above mentioned publication the areas with possible eigenparameters were firmly given. In the second publication the areas (patches) were selected from experience and also were done.

Unit impulses of subdomain-wise uniformly distributed eigenparameters (generally linear or higher order polynomials) enable one to find material properties from comparison of results from experimental studies and numerical analysis at selected points, as was done in other papers of the author of this paper on geomechanical problems, [3, 4], for example. The only problem still occurring is: find the optimal distribution of patches (subregions), where eigenstrains are introduced or considered. This is a problem of combined optimization, where the principal variables depend on subdomains (patches), the uniform distribution (this is one of possible approximations) of eigenparameters is assumed. This problem is not easy to solve, as the optimization of principal variables must be iterated, and a reasonable tool for it should be found. One such tool can serve Inverse variational principles, which hold the volume of the domain as constant, and design variables are subdomains, or their representatives, [5].

\section{Transformation field analysis}

In this section the general procedure for coupled modeling on the firm patches is briefly introduced using the TFA. It may be done in terms of many modern numerical methods. First, let us consider that the body $\Omega$ under consideration (part of a structure, element, and system of more elements, composite, in our case rock surrounding tunnel) behaves linearly, i.e. Hooke's linear law is valid in the entire body. When the problem is correctly posed, the displacement vector, strain and stress tensors can be obtained from the Navier equations, kinematical equations, and linear Hooke's law.

In the second step we select points, where the measured values are available, either from experiments in laboratory, or from "in situ" measurements. We also select points $A_{r}$, or disjoint regions (subdomains) $\Omega_{r}, r=1, \ldots, n$, from the body under study, and apply there successively unit eigenparameter impulses (either eigenstresses or eigenstrains) to get an influence tensors (matrices). Moreover, let the set of points where the measured values are known be $B_{s}, s=1, \ldots, m$. Then the real stress $(\sigma)^{s}$ at $B_{s}$ is a linear hull of stress $\left(\sigma^{\text {ext }}\right)^{s}$ at $B_{s}$ due to external loading and eigenstrains $(\mu)^{r}$ and $\left(\varepsilon^{\mathrm{pl}}\right)^{r}$, or eigenstress $(\lambda)^{r}$ and relaxation stress $\left(\sigma^{\text {rel }}\right)^{r}$ at $A_{r}$ (similar relations are valid for overall strain field $\varepsilon$ or displacements $u)$ :

$$
\left(\sigma_{i}\right)^{s}=\left(\sigma_{i}^{\mathrm{ext}}\right)^{s}+\left(P_{i k}^{\sigma}\right)^{s r}\left(\mu_{k}\right)^{r}+\left(Q_{i k}^{\sigma}\right)^{s r}\left(\varepsilon_{k}^{\mathrm{pl}}\right)^{r},
$$

or

$$
\left(\sigma_{i}\right)^{s}=\left(\sigma_{i}^{\mathrm{ext}}\right)^{s}+\left(R_{i k}^{\sigma}\right)^{s r}\left(\lambda_{k}\right)^{r}+\left(T_{i k}^{\sigma}\right)^{s r}\left(\sigma_{k}^{\mathrm{rel}}\right)^{r}
$$




$$
\left(u_{i}\right)^{s}=\left(u_{i}^{\mathrm{ext}}\right)^{s}+\left(P_{i k}^{u}\right)^{s r}\left(\mu_{k}\right)^{r}+\left(Q_{i k}^{u}\right)^{s r}\left(\varepsilon_{k}^{\mathrm{pl}}\right)^{r},
$$

or

$$
\left(u_{i}\right)^{s}=\left(u_{i}^{\mathrm{ext}}\right)^{s}+\left(R_{i k}^{u}\right)^{s r}\left(\lambda_{k}\right)^{r}+\left(T_{i k}^{u}\right)^{s r}\left(\sigma_{k}^{\mathrm{rel}}\right)^{r}
$$

where the Einstein summation rule has been used and the influence tensors $P, Q$, and also $R$ and $T$ may be identical, as any eigenparameter may stand for the plastic or relaxation parameter (say, eigenstrain may stand for plastic strain, which is obvious from (1) and (2)). The strain and stress components are written in vector form. Note that $\lambda=-L \mu$ holds, where $L$ is the elastic stiffness tensor.

The first relations in (1) and (2) describe the initial strain method while the second relations in those equations formulate the initial stress method.

On the other hand measured stresses $\left(\sigma_{i}^{\text {meas }}\right)^{s}$, or measured displacements $\left(u_{i}^{\text {meas }}\right)^{s}$ are available in a discrete set of points. A natural requirement is formulated in terms of steepest descent type "error functionals" $I$, which express that the values of measured and computed values be as close as possible:

$$
I_{\sigma}\left[\left(\mu_{k}\right)^{r}\right]=\sum_{i=1}^{6} \sum_{s=1}^{m}\left[\left(\sigma_{i}\right)^{s}-\left(\sigma_{i}^{\text {meas }}\right)^{s}\right]^{2} \rightarrow \text { minimum }
$$

or

$$
I_{u}\left[\left(\mu_{k}\right)^{r}\right]=\sum_{i=1}^{6} \sum_{s=1}^{m}\left[\left(u_{i}\right)^{s}-\left(u_{i}^{\text {meas }}\right)^{s}\right]^{2} \rightarrow \text { minimum }
$$

where the sum is taken over $i$ and $s$. Differentiating $I$ by $\left(\mu_{\alpha}\right)^{\beta}$ yields a linear system of equations for $\left(\mu_{j}\right)^{1}$ :

$$
\left(A_{\alpha k}\right)^{\beta r}\left(\mu_{k}\right)^{r}=Y_{\alpha}^{\beta}, \alpha=1, \ldots, 6, \beta=1, \ldots, m,
$$

where

$$
\begin{gathered}
\left(A_{\alpha k}\right)^{\beta r}=\sum_{i=1}^{6} \sum_{s=1}^{m}\left(P_{i k}^{\sigma}\right)^{s r}\left(P_{i \alpha}^{\sigma}\right)^{s \beta} \\
Y_{\alpha}^{\beta}=\sum_{i=1}^{6} \sum_{s=1}^{m}\left[\left(\sigma_{i}^{\text {meas }}\right)^{s}-\left(\sigma_{i}^{\text {ext }}\right)^{s}-\left(Q_{i k}^{\sigma}\right)^{s r}\left(\varepsilon_{k}^{\mathrm{pl}}\right)^{r}\right]\left(P_{i \alpha}^{\sigma}\right)^{s \beta}
\end{gathered}
$$

in the case (3), and

$$
\left(A_{\alpha k}\right)^{\beta r}=\sum_{i=1}^{6} \sum_{s=1}^{m}\left(P_{i k}^{u}\right)^{s r}\left(P_{i \alpha}^{u}\right)^{s \beta}
$$




$$
Y_{\alpha}^{\beta}=\sum_{i=1}^{6} \sum_{s=1}^{m}\left[\left(u_{i}^{\mathrm{meas}}\right)^{s}-\left(u_{i}^{\mathrm{ext}}\right)^{s}-\left(Q_{i k}^{u}\right)^{s r}\left(\varepsilon_{k}^{\mathrm{pl}}\right)^{r}\right]\left(P_{i \alpha}^{u}\right)^{s \beta}
$$

in the case (4). Recall that the patches are selected as fixed, their position and shape are firmly given.

\section{Nonlinear law given}

The previous section was devoted to application of the TFA to coupled modeling assuming the starting (initial) stage of computation is linear elastic. This case is appropriate for large scale problems, involving plasticity, hereditary problems and some softening rules. On the other hand, probably a more precise approach would be to start with the plastic law, at least approximately.

It is well known that some cases of softening lead to multivalued functions. The procedure described in the previous section cannot include this case. If the initial stage is described by a certain plastic rule, the larger scale of materially nonlinear problems can be solved in similar way as before. Let us concentrate on such problems.

If a certain plasticity law is proposed we can write using the splitting of elastic and plastic influences the resulting stresses and displacements as:

$$
\left(\sigma_{i}\right)^{s}=\left(\sigma_{i}^{\mathrm{ext}}\right)^{s}+\left(Q_{i k}^{\sigma}\right)^{s r}\left(\varepsilon_{k}^{\mathrm{pl}}\right)^{r}
$$

or

$$
\begin{aligned}
& \left(\sigma_{i}\right)^{s}=\left(\sigma_{i}^{\mathrm{ext}}\right)^{s}+\left(T_{i k}^{\sigma}\right)^{s r}\left(\sigma_{k}^{\mathrm{rel}}\right)^{r} \\
& \left(u_{i}\right)^{s}=\left(u_{i}^{\mathrm{ext}}\right)^{s}+\left(Q_{i k}^{u}\right)^{s r}\left(\varepsilon_{k}^{\mathrm{pl}}\right)^{r},
\end{aligned}
$$

or

$$
\left(u_{i}\right)^{s}=\left(u_{i}^{\mathrm{ext}}\right)^{s}+\left(T_{i k}^{u}\right)^{s r}\left(\sigma_{k}^{\mathrm{rel}}\right)^{r}
$$

From the above equations it immediately follows that, for example,

$$
\left(\sigma_{i}\right)^{s}=\left(S_{i k}^{\sigma}\right)^{s}+\left(P_{i k}^{\sigma}\right)^{s r}\left(\mu_{k}\right)^{r},\left(u_{i}\right)^{s}=\left(S_{i k}^{u}\right)^{s}+\left(P_{i k}^{u}\right)^{s r}\left(\mu_{k}\right)^{r}
$$

holds, i.e. the influence of elasticity and plasticity is hidden in the first terms of the right hand sides of (8). From (8) two possibilities obviously appear: Either plastic effects disappear in the first terms of the r.h.s. of (8) or they are considered there. Certain starting plastic rules involved in (8) are discussed in [4].

Using the expressions (8) and the minimum conditions (3) or (4), the conditions for a minimum are obtained from (5) with

$$
Y_{\alpha}^{\beta}=\sum_{i=1}^{6} \sum_{s=1}^{m}\left[\left(\sigma_{i}^{\text {meas }}\right)^{s}-\left(\sigma_{i}^{\text {ext }}\right)^{s}\right]\left(P_{i \alpha}^{\sigma}\right)^{s \beta}
$$




$$
Y_{\alpha}^{\beta}=\sum_{i=1}^{6} \sum_{s=1}^{m}\left[\left(u_{i}^{\text {meas }}\right)^{s}-\left(u_{i}^{\text {ext }}\right)^{s}\right]\left(P_{i \alpha}^{u}\right)^{s \beta}
$$

The left hand sides hold their former expression.

\section{Inverse variational principles}

Following extended primary variational principles one can write the energy functionals on the whole domain $\Omega$ as:

$$
\Pi=\frac{1}{2} a(u, u)-[p, u] \rightarrow \text { minimum }
$$

where $a(.,$.$) is an energetic norm and [.,.] is the scalar product on the boundary \Gamma$ of $\Omega$, with $p$ being prescribed tractions. Let us divide $\Omega$ into $m$ disjoint subregions (patches) $\Omega_{r}$, union of them is $\Omega$. Then (9) can be rewritten as

$$
\Pi=\sum_{r=1}^{m} \frac{1}{2} a_{r}(u, u)-[p, u] \rightarrow \text { minimum }
$$

where $a_{r}(.,$.$) is an energetic norm on each \Omega_{r}$. Then the problem appears not to be properly defined. In order to ensure that the problem is correctly posed, the volumes (or areas in 2D) have to be bounded and their measure has to be given. The functional (9) then is improved as:

$$
\begin{array}{r}
\Pi(u, \Omega)=\sum\left[\frac{1}{2} a_{r}(u, u)+\omega_{r}\left(\int \Omega \mathrm{d} \Omega_{r}-C_{r}\right)\right]-[p, u] \rightarrow \\
\rightarrow \text { stationary }
\end{array}
$$

where $C_{r}$ is a measure of $\Omega_{r}$, see [5], and $\omega_{r}$ are the lagrangian multipliers. The internal energy is a sum of integrals over appropriate domains $\Omega_{r}$ of potentials $(W)^{r}$, which in our case reads as:

$$
(W)^{r}=\left(\sigma_{i}\right)^{r}\left[\left(\varepsilon_{i}\right)^{r}-\left(\mu_{i}\right)^{r}\right]
$$

where $\left(\mu_{i}\right)^{r}$ has been considered uniformly distributed in each $\Omega_{r}$. Hence, Euler's equations follow as:

1. Variation by displacements yields equilibrium equations involving partially uniformly distributed eigenstrains $\left(\mu_{i}\right)^{r}$. They are given from (5) for a supposed distribution (shape) of $\Omega_{r}$. 
2. Approximating the problem in the sense of FEM with $K$, say, then the changes in the fields with respect to the subdomains can formally be written as:

$$
\frac{1}{2} \frac{\partial K}{\partial p_{i}} U_{i} U_{k}+\omega_{r} \frac{\partial \Omega_{r}}{\partial p_{i}}=0
$$

where $p_{r}$ are internal parameters declaring the shape of the subdomain $\Omega_{r}$. From (10) it immediately follows that the lagrangian multipliers have to be constant for each $r$, i.e. on each subdomain $\Omega_{r}$ all components of eigenstrain tensor remain uniform.

3. Partial differentiation of (8) by $\omega_{r}$ ensures that the measures of the subdomains are unchanged. Some recommendations on how to introduce the internal shape parameters could be found in [5], for example.

\section{Numerical procedure for two subdomains}

To show the ability of the above submitted procedure consider a simply supported steel fiber reinforced concrete beam with the length of $30 \mathrm{~cm}$, the height of $12 \mathrm{~cm}$, the bending stiffness $I=1440 \mathrm{~cm}^{4}, E=2 \mathrm{GPa}$. The width is 10 $\mathrm{cm}$. The problem is solved as a stretched plate, the shape of the external boundary of which remains constant and one patch is considered. Concentrated forces are applied at one third and two thirds of the span, i.e. four points test is studied. The symmetric case is solved, shear eigenstrains are neglected, and transversal eigenstrain disappears, too. The force $\mathrm{F}$ applied is $4 \mathrm{kN}$, distributed along the width, so that the stress in the elastic state is $1.67 \mathrm{MPa}$.

Measured values of deflections are as follows: under the force at the lower edge of the beam it was $0.69 \mathrm{~cm}$, at the middle of the span $0.828 \mathrm{~cm}$. The initial stage is considered elastic, plasticity occurs due to eigenparameters, which stand here for plastic strains.

For the optimized shape of the patch we use condition (4). Note that the number of unknown eigenparameters cannot exceed the number of measured values. This must be understood in such a way that one component of the eigenparameter tensor is one unknown. For example, if we take into consideration a full 2D tensor, than four components create the unknowns in one patch. Consequently, for one patch we need at least five measured values of either displacement, or stresses, or both, as obviously the conditions (3) and (4) can be combined.

The strain at the middle of the span in the lower edge was calculated as 0.000835 , while the resulting strain dropped to the value of 0.000738 , i.e. the eigenstrain lowered the strain by approximately 0.0001 . The movement of the boundary of the optimal patch undergoes criteria, which are described in the second example, which is more general.

The final situation is described in Fig. 1, where the shape of the uniformly distributed eigenstrains is shown (we consider only one component of the eigenstrain tensor, namely the horizontal eigenstrain, according to our above mentioned assumption). 


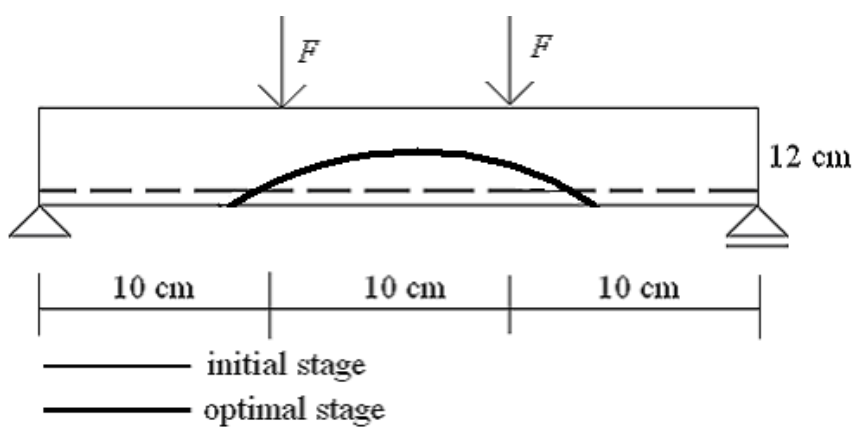

Figure 1: $\quad$ Four point test on a beam with one patch.

The second example is much more complicated and shows us how to use the procedure in underground structure assessment, namely for description of behavior of the rock surrounding the tunnel lining. Also here, one patch is considered for simplicity. As in the eigenparameters also the influence of purchase can be involved, the problem of loading of the lining is solved as a $2 \mathrm{D}$ problem.

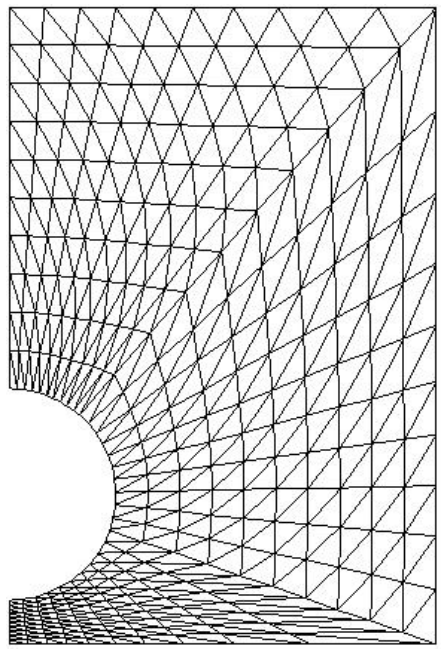

a)

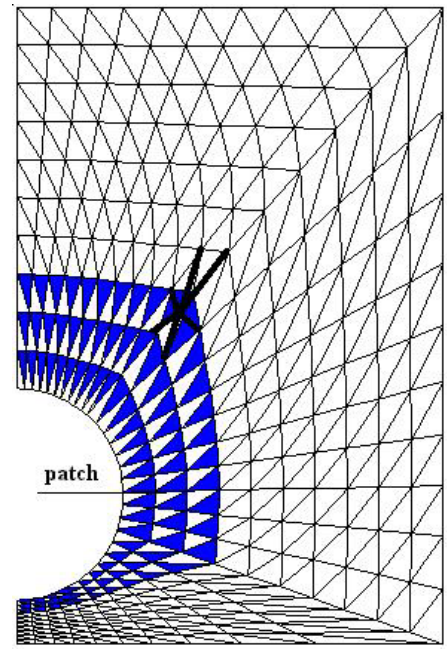

b)

Figure 2: a) Starting triangular mesh and b) highlighted domain describes the patch considered.

The height of the domain, in which the problem is solved, is $30 \mathrm{~m}$, the diameter of the tunnel is $10 \mathrm{~m}$, the distance of the axis of the tunnel from the lower boundary of the domain is $15 \mathrm{~m}$, and the width of the model is $15 \mathrm{~m}$. A triangular mesh is depicted in Fig. 2a), and the starting shape of the patch is displayed in Fig. 2b). In these pictures as well as in the next pictures no tunnel lining is depicted in order not to disturb the contour lines (hypsography). 
The problem starts with the solution of the plastic stage obeying the generalized Mohr-Coulomb hypothesis with the following material parameters:

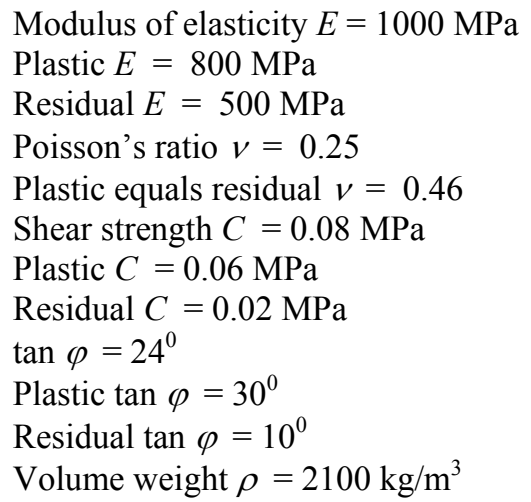

where $\varphi$ is the angle of internal friction.

Using the above said parameters describing material properties, which correspond with the class $\mathrm{R}_{3}$ of the rock according to geological standards. The tunnel lining is made from concrete and no plastic behavior is assumed. After computing the plastic state with the above parameters, the initial stage is created and the eigenstrains can be introduced. The initial shape of the patch is depicted by the highlighted subdomain in Fig. 2b). In this figure also possible movement of one nodal point is illustrated by thick lines. Parameters $p_{i}$ are distances of the patch boundary nodal points from the center of the tunnel in our case. Movement of each point causes also a change in the mesh, as is obvious from Fig. 2b).

The measured values were also vertical deflections on the contact of the lining and rock. The values were taken from a scale model built up in stands, as described in [3], for example. There were nine measurement points along the lining; the values at symmetric points were averaged.

In Fig. 3a) hypsography of vertical stresses in the starting plastic stage is depicted and in Fig. 3b) hypsography of vertical stresses in the final stage after optimization of the shape of patch is displayed. The difference between these two pictures is not as distinct as supposed, the plastic law and the material properties were selected in a good way, and the influence of the purchase is not too important. From this assertion immediately follows the conclusion that the eigenstrains can be also considered as a measure of error of the plastic law selected. On the other hand, if the choice of the plastic law is wrong, the initial stage starts with elasticity, for instance, as was the case of the previous example, more accurate results can be expected only if more patches are selected, not only one, as in our examples.

In Fig. 4a), vertical displacements, which were compared with the measurements on the scale model, are again drawn in the form of hypsography. Important is the fact that at one of the measured points, at point $\mathrm{A}$, the measured vertical displacement was $4.12 \mathrm{~cm}$, so that the results are very versatile. For completeness in Fig. 4b) the shape of the patch in the final stage is also shown. 


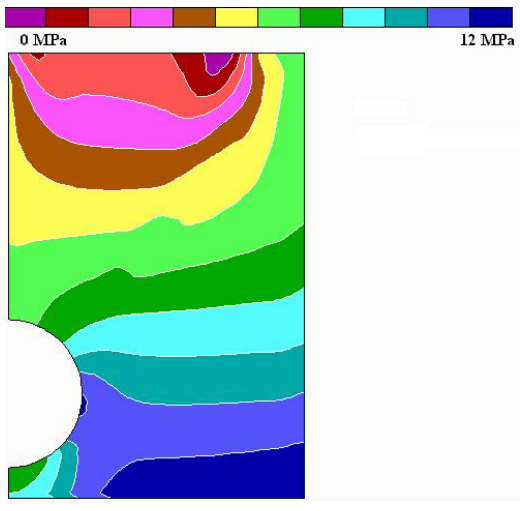

a)

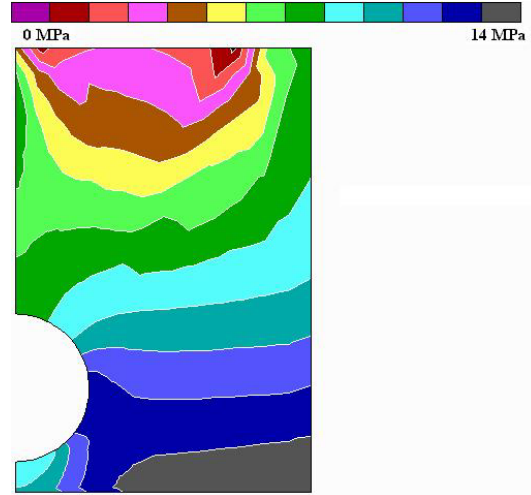

b)

Figure 3: Vertical stresses in a) plastic stage and b) after optimization.

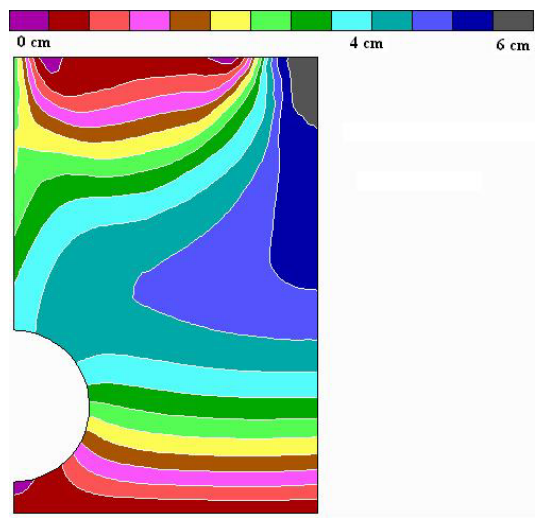

a)

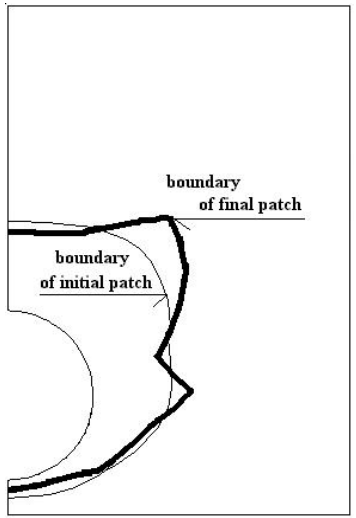

b)

Figure 4: a) final displacements and b) shape of the optimal patch.

\section{Conclusions}

In some previous papers of the author it has been shown that the coupled numerical and experimental (scale) modeling or the on site measurements can basically improve identification of a numerical mechanical model. The only problem appeared the choice of subdomains (patches). No solution has been proposed so far. This paper tries to improve this lack of information using Inverse variational principles. Although simple examples are presented here, the generalization to more patches is straightforward.

It is worth noting that for subdomains of large extent an extensive number of measurements are necessary. In former papers three or at most four subregions have been considered in applications to underground structures, particularly for 
assessment of tunnel face stability. The reason consists of the fact that (5) is generally created for 3 in 2D or 6 in 3D components of eigenstrains (or eigenstresses), which means it is necessary to determine at least 24 unknown eigenparameters in 3D for four subdomains in each iterative step. From the point of view of numerical analysis this does not cause any problem, but to feed (5) at least by 25 measurements the original domain $\Omega$ can lead to quite a complicated problem or an insufficient set of data.

\section{Acknowledgements}

The financial support of GAČR, project number 103/06/1124 is appreciated. The research has also been supported by a grant of Ministry of Education of the Czech Republic number MSM6840770001.

\section{References}

[1] G. J. Dvorak, P. P. Prochazka: Thick-walled composite cylinders with optimal fiber prestress, Composites Part B 27B, 643-649, 1996

[2] P. P. Procházka, A. E. Yiakoumi: Nonlinear transformation field analysis of inelastic composite structures, In Proc. Conference on Composites/Nano Engineering, Colorado, Univ. of New Orleans, CD, 2.7.-8.7. 2006.

[3] P. P. Procházka, J. Trčková: Coupled Modeling of Concrete Tunnel Lining, In Proc. Our World in Concrete and Structures, Singapore, 2000, 125-132.

[4] P. P. Procházka, J. Trčková: Assessment and Control of Tunnel Structures based on coupled modeling. Submitted to Géotechnique.

[5] P. P. Procházka: Shape optimal design using Inverse Variational Principles. Submitted to EABE. 